\title{
La reacción conservadora. Algunas preguntas teóricas en torno al debate sobre el plural del español y la aparición de la " $\mathrm{e}$ " como práctica de lenguaje inclusivo en Argentina
}

\author{
Marina Cardelli (UBA) ${ }^{1}$ \\ Universidad de Buenos Aires, Argentina \\ mcmarinacardelli@gmai.com
}

La charla fue hace un tiempo y el caballero tenía argumentos gramaticales. "Presidente" -decía el señor-es como "cantante". Y -decía- aunque parece un sustantivo es otro tipo de palabra, un participio presente, o lo que quedó de los participios presentes del latín. Una palabra que señala a quien hace la acción: quien preside, quien canta. Justamente, no tiene género. "¿Vas a decir la cantanta?"

En la mesa corrió el silencio de la duda. Fue un instante y lo cortó la voz decidida de la escritora Claudia Piñeiro: “ ¿Y 'sirvienta' tampoco decís? ¿O 'presidenta' no pero 'sirvienta' sí”? Gol.

Kolesnikob, Patricia, Clarín, 20/12/2019

En la palabra se ponen en funcionamiento los innumerables hilos ideológicos que traspasan todas las zonas de la comunicación social. Por eso es lógico que la palabra sea el indicador más sensible de las transformaciones sociales, inclusive aquellas que apenas van madurando, aquellas que aún no se constituyen nuevamente ni encuentran acceso todavía a los sistemas ideológicos ya formados y consolidados. La palabra es el medio en el que se acumulan lentamente aquellos cambios cuantitativos que aún no logran pasar a una nueva cualidad ideológica, ni dar origen a una nueva y acabada forma ideológica. La palabra es capaz de registrar todas las formas transitorias, imperceptibles y fugaces de las transformaciones sociales.

Voloshinov, Valentín, El Marxismo y la Filosofía del Lenguaje, 1929

\section{Introducción}

Por primera vez en la historia, el 13 de Junio de 2018 se debatió en la Cámara de Diputados ${ }^{2}$ de la República Argentina el proyecto de Ley de Interrupción Voluntaria del Embarazo, luego de siete años consecutivos de ser presentado por la Campaña Nacional por el Derecho al Aborto Legal Seguro y Gratuito ${ }^{3}$ y convocó a un millón de personas en la puerta del Congreso Nacional. Ese fenómeno trajo consigo el uso masivo del pañuelo verde (insignia de la

1 Marina Cardelli es Profesora en Letras de la Facultad de Filosofía y Letras de la UBA y becaria doctoral del Consejo Nacional de Investigaciones Científicas y Técnicas de Argentina (CONICET). Es docente de la cátedra de Semiología en el Ciclo Básico Común de la UBA y de Estudios del Discurso en la UNSAM. Asimismo, dicta talleres de lectura y escritura en la UNA. Participa en proyectos de investigación (UBACyT, ANPCyT) que abordan temáticas vinculadas a los estudios del lenguaje y el discurso desde una perspectiva glotopolítica.

2 Este ensayo está destinado a todos: mujeres cis, lesbianas, travestis, trans, intersex, queer, varones cis y cualquier otra identidad de género. Sin embargo, se utilizará el masculino como forma plural inclusiva para designar a más de una persona (a menos que sean todas mujeres cis) y para todas las formas genéricas -en el sentido de universales, como "el Hombre"-. Se resaltará distintivamente la palabra completa cada vez que eso ocurra, con el objetivo de que todos los que se sienten incluidos tengan la oportunidad de identificar cada vez que eso no les ocurre a muchos otros.

3 La fecha prevista para el tratamiento en la Cámara de Senadores es el 8 de Julio de 2018, por lo que es posible que para la fecha de publicación del presente ensayo el aborto sea legal en la República Argentina. 
Campaña Nacional), convertido en un emblema nacional de la lucha feminista y actualmente en expansión en el resto de los países de América Latina. Los estudiantes secundarios fueron uno de los sectores más activos en todas esas luchas y empezaron a visibilizar en los grandes medios de comunicación una práctica de lenguaje inclusivo que ya tenía bastante tiempo al interior de los ámbitos feministas: el uso de " $\mathrm{e}$ " como morfema del masculino inclusivo, en remplazo de " $\mathrm{o}$ ".

La masividad y el alcance internacional de la lucha feminista en los últimos años que, lejos de sostener una agenda sectorial, es uno de los actores más dinámicos de la lucha antineoliberal -y anticapitalista- en el mundo (Minici, 2018) es el contexto en el cual se desarrolló este proceso de movilización masiva.

Una de las declaraciones públicas que más trascendió y que lo convirtió en un debate de alcance nacional fue la de una adolescente que opinaba sobre la acción de los estudiantes secundarios durante el debate parlamentario del aborto:

“Desde ya digo que estamos comunicándonos con toda la comunidad educativa, les padres, les docentes, para ver, por ejemplo, las clases de apoyo del curso de ingreso, si algunes docentes quieren venir, incluso en el medio de la toma, a dar clases, está la posibilidad porque el reclamo no es contra la institución particularmente, sino para visibilizar un reclamo que estamos teniendo les estudiantes que es que salga el proyecto de ley. Hay poques diputades que están indecises, y queremos demostrarles que a nosotres no nos va a pasar por el lado que sigan muriendo mujeres, o que decidan frenar eso e ilegalizar el aborto"

Estudiante secundaria argentina en una toma de escuela, 12 de junio de 2018, un día antes del tratamiento de la Ley de Interrupción Voluntaria del Embarazo, para escuchar:

h t t p s:// w w w . you tube.com/ watch? $v=$ IwozaE24z_w
Cuando esa entrevista se hizo pública, el debate no tardó en crecer, al día siguiente varios diarios de alcance nacional publicaron notas de opinión, editoriales, chistes etc.; empezó a hablarse del tema tanto en los medios masivos de comunicación como en las aulas y salas de profesores. Los comentarios de la publicación en las redes sociales son elocuentes respecto de la orientación general de las opiniones que empezaron a circular:

1. "señora sa hija ha nacide can retrases mentales severes"

2. "Pobre chica, me agarra pena. Perdón, fui poco inclusivo. Pobre chique, me agarra el pene"

3. "Habla bien la concha de tu puta madre anteojuda incogibleee!!"

4. "hacen falta más Barredas 4 para evitar esto"

5. "Deja de arruinar mi hermoso idioma, retrasada. Por que no usas la educación para estudiar en lugar de adoctrinarte?"

\section{6. "Los de la RAE se quieren pegar un tiro:v"}

En los ejemplos 2, 3 y 4 la respuesta tiene elementos de violencia machista: amenaza encubierta, insulto misógino ("concha de tu puta madre" "incogible") y acoso. En los casos 3,4 y 5 hay una representación lingüística asociada al valor de preservar "La Lengua" de quién la "arruina", de quien la habla "mal" y un reconocimiento de la institución supuestamente encargada de su cuidado, la Real Academia Española. Los casos 1 y 5 muestran la aparición de otra representación, asociada, por un lado a la anormalidad (apartamiento de la norma "retrases mentales severes") y, por el otro, a que eso reciba una sanción social -por ejemplo, el insulto-. Los comentarios particulares no son el objeto de reflexión de este texto y han sido seleccionados al azar, sin embargo, es importante identificar qué reacciones suscita en la sociedad esta intervención colectiva sobre la lengua: violencia e indignación. Los comentarios de docentes y padres en las escuelas no mostraron una matriz diferente.

4 Ricardo Alberto Barreda es un odontólogo argentino que en 1992 asesino a su esposa, sus dos hijas y su suegra. En 1995 fue condenado a prisión perpetua. 
El "lenguaje inclusivo" o el "sexismo en la lengua" no son temas nuevos y hace varios años que se debaten, tanto en el ámbito de los estudios del lenguaje y el discurso, como en los ámbitos feministas. Se han expresado en contra con mucho ímpetu desde instituciones que asumen la tarea de "preservar el idioma", con tanto éxito que-como pudimos ver- la ideología de la preservación es dominante, está completamente naturalizada en los medios, en las calles, además de las academias.

Merece atención y reflexión que una porción muy numerosa de estudiantes secundarios viven su vida cotidiana utilizando el morfema " $\mathrm{e}$ ", que ya no se limita a una propuesta para la escritura, como lo son la " $x$ " o el “@”, sino de un salto a la oralidad que supone, no solamente algunas expresiones de gramaticalidad discutible, como otras versiones de lenguaje inclusivo, sino una puesta en discusión de todo el sistema de flexión genérica en el español. Ese proceso no tiene ninguna estabilidad y su uso no es homogéneo ni sistemático. Tampoco es único en el mundo, ya que en muchos países el crecimiento del feminismo como movimiento político está suscitando propuestas similares. En el caso argentino $-\mathrm{y}$, podríamos decir, de las grandes ciudades de Argentina- los usos son, al menos, dos: en algunos contextos " $\mathrm{e}$ " reemplaza el uso de "o" como morfema de masculino inclusivo de todos los géneros: "les compañeres"; pero en otros se suma para nombrar a aquellas personas con identidades que no coinciden con los géneros binarios tradicionales -femenino o masculino-: "compañeros, compañeras y compañeres". Este fenómeno tiene larga data en los ámbitos feministas, en especial la comunidad de la diversidad sexual, ya que no es simplemente una ocurrencia gramatical, sino que se desarrolló junto al crecimiento en visibilidad política de esas identidades.

El mismo conocimiento público adquirieron otras formulaciones típicas de los ámbitos feministas en proceso de debate parlamentario sobre el aborto, como referirse a "personas gestantes" y no a "mujeres" para dejar sentado que existen personas que abortan y no se reconocen como mujeres. Es decir, el proceso de popularización de nuevas propuestas de lenguaje inclusivo fue el resultado del crecimiento de un sujeto político del feminismo que ya no eran sólo las mujeres, sino otras identidades.

Esteensayo intenta poner sobrela mesa algunas dimensiones relevantes y poco consideradas hasta ahora desde los estudios lingüísticos y discursivos en lo que refiere al uso del morfema " $\mathrm{e}$ ". Lejos de asumir una posición neutra, o que pueda "resolver" algún tipo de "incordio" sobre la base de lo que resulta "adecuado" o "correcto" para una perspectiva prescriptiva de la lengua como entidad abstracta, consideramos que estamos ante un debate que reviste la forma de lo político y exige, por ello, una abordaje glotopolítico que, aunque se localiza a nivel del sistema, no puede abordarse al margen de las prácticas lingüísticas y discursivas.

Muchas veces lo más importante no son las respuestas, sino las preguntas. Una pregunta presupone un objeto de reflexión, una posición teórico-política y un objetivo de conocimiento. Si no estamos de acuerdo en cuál es, en este caso, el objeto de reflexión, la posición teórico-política y el objetivo de conocimiento, no es posible avanzar en debates conducentes. Hasta hoy este debate ha tenido, muchas veces, el rasgo de darse sin acuerdo en esas dimensiones básicas $\mathrm{y}$, de algún modo, ha clausurado el necesario -o urgente- debate al interior del campo de los estudios del discurso acerca de qué rol vamos a tener los especialistas y desde qué posiciones teórico-políticas vamos hablar.

Una afirmación de consenso entre la mayoría de las posiciones teóricas es que la Lengua es social, presunción sin la cual este debate estaría cerrado. En un extremo de tales posiciones están las teorías del reflejo, es decir, aquellas que consideran que la lengua refleja lo que ocurre en la sociedad -desde una concepción determinista y lineal de la relación entre la estructura social y la superestructura- y que para terminar con el androcentrismo y el sexismo en la lengua habrá que terminar primero con el de la sociedad. En el otro extremo, están las posiciones más radicalizadas del llamado relativismo lingüístico (la llamada "hipótesis de Sapir- 
Whorf"), de base saussuriana, que sostienen que la Lengua configura las categorías desde las cuales pensamos el mundo, hasta un punto tan extremo que una lengua es una visión del mundo irreductible a otra y que, en algún punto, aquellos significados que no están en la lengua no pueden pensarse. Desde el inicio vamos a dejar planteado que esas dos posiciones dejan afuera la densidad del debate que, desde nuestro punto de vista reside en preguntarse de qué modo participa el lenguaje en la reproducción de relaciones de dominación económica y política para ser susceptible de intervenciones políticas por parte de sectores de poder (o resistencia). Esta pregunta reenvía hacia, por un lado la configuración histórica de la lingüística moderna sobre la base de la dicotomía lengua/ habla que este debate pone en cuestión; por otro lado, las lingüistas feministas, que han dado cuenta del carácter androcéntrico y sexista del lenguaje y que son sistemáticamente ignoradas o silenciadas en los ámbitos disciplinares; por último, señala hacia la presencia creciente de un sujeto político que se enfrenta a las relaciones de dominación económica, política y cultural $y$ que se despliega, incluso, en intervenciones concretas sobre las prácticas lingüísticas que nos obligan a dar cuenta que una política: la política de los feminismos sobre el lenguaje ${ }^{5}$.

\section{El problema glotopolítico y las miradas feministas de las relaciones de dominación}

El término Glotopolítica se refiere a las distintas formas en que las acciones sobre el lenguaje participan en la reproducción o transformación de las relaciones de poder. La perspectiva de la glotopolítica contemporánea (Marcellesi y Guespin 1986, Arnoux 2000 y 2008, Laroussi 2003) estudia los diversos tipos de intervenciones en el espacio público del lenguaje y el modo en que participan en la conformación, reproducción o transformación de las relaciones sociales y de las estructuras de poder, en el ámbito local, nacional, regional o mundial. Es decir, que tiene como objetivo indagar en diversos tipos de discursos sociales que toman la lengua como objeto de estudio, de reflexión y/o de valoración -gramáticas, diccionarios, ensayos, teorías lingüísticas, etc. (Arnoux y Luis 2003)

Una de las tareas fundamentales de la glotopolítica ha sido identificar que existen ideologías lingüísticas que orientan, en cada época, de una manera bastante restrictiva, la evaluación de las situaciones lingüísticas:

Las ideologías lingüísticas son sistemas de ideas que articulan nociones del lenguaje, las lenguas, el habla y/ o la comunicación con formaciones culturales, políticas o sociales específicas. Aunque pertenecen al ámbito de las ideas y se pueden concebir como marcos cognitivos queligan coherentemente el lenguaje con un orden extralingüístico, naturalizándolo y normalizándolo, también hay que señalar que se producen y reproducen en el ámbito material de las prácticas lingüísticas y metalingüísticas, de entre las cueles presentan para nosotros interés especial las que exhiben un alto grado de institucionalización (Del Valle 2007).

En el caso de los debates acerca de lenguaje inclusivo -como puede verse en los comentarios que recibió el video- los condensados ideológicos que sostienen ciertas posiciones se articulan de forma muy particular con discursos visiblemente machistas y misóginos. De modo tal que no es posible impulsar un debate serio respecto del lenguaje inclusivo en general, y del uso de " $\mathrm{e}$ " en particular sin asumir alguna posición teórica respecto las propuestas -ya abundantes, a esta altura- de las teorías feministas acerca de la construcción social del género, del vínculo que entabla con las relaciones de desigualdad y violencia y la estructuración profunda que tiene con la estructura social de producción y reproducción.

Para la glotopolítica, en el lenguaje se afirman y negocian las identidades sociales de los

5 Hablamos de "feminismos" en plural porque existen muchas corrientes teóricas y políticas que se asumen desde una posición feminista que no tienen acuerdo en sus bases teóricas ni en su acción política. 
interlocutores. Existen regímenes de normatividad que, en el seno de esa colectividad concreta, articulan la relación entre las formas lingüísticas y su valor social, es decir, que los regímenes de normatividad son las relaciones de dominación política que yacen tras las ideologías lingüísticas. Arnoux y Del Valle (2010) sostienen lo siguiente acerca de tales regímenes:

Partamos del hecho de que toda colectividad humana se caracteriza por la existencia de ciertas condiciones sociales y relaciones de poder. Son estos factores contextuales los que estructuran el mercado lingüístico, es decir, el régimen de normatividad o sistema que asigne valores diferentes a los usos del lenguaje. El lugar que un individuo ocupe en la sociedad, los espacios a los que tenga acceso y la capacidad que posea para negociar su rol en ese entorno determinar su predisposición a actuar de una cierta manera o a valorar de uno $\mathrm{u}$ otro modo las acciones de otros. El individuo desarrolla, en terminología de Pierre Bourdieu, un habitus. Estará, por tanto, en disposición de usar una $o$ varias lenguas, una $u$ otra lengua, una $u$ otra variedad de una lengua, dependiendo de su ubicación y capacidad de maniobra en el complejo entramado social. Y de esta misma posición, y por tanto de su grado de sometimiento o autonomía con respecto al régimen de normatividad imperante, dependerá su imposición de valorar de un modo u otro el espectro de prácticas lingüísticas que se encuentre (Arnoux, Del Valle 2010).

El orden simbólico que imponen las clases dominantes incluye esquemas de percepción y evaluación de las normas lingüísticas y de dispositivos normativos tendientes a orientar prácticas y creencias. Es decir que esas ideologías lingüísticas que pudimos esbozar más arriba integran sistemas ideológicos más amplios y se ponen al servicio de ellos (Arnoux 2008). Analizar las condiciones sociales y relaciones de poder que estructuran los regímenes de normatividad vigente supone acudir a las teorías feministas que han pensado la problemática de la dominación de forma integral. Por un lado, existen estudios que, desde el feminismo, piensan el sistema capitalista y su funcionamiento como sistema de dominación; por otro, están los estudios de género, que han pensado a lo largo de las últimas décadas el modo en el que se configuran las relaciones de poder sobre la base de la diferencia sexual.

Una de las autoras que intentó repensar el desarrollo del modo de producción capitalista desde una perspectiva feminista es Silvia Federici (2004), recuperando la caza de brujas medieval como un elemento central de su surgimiento. Sostiene que la llamada "acumulación originaria" supuso una nueva división sexual del trabajo en la cual la función reproductiva de las mujeres está destinada a la reproducción de la fuerza de trabajo. En el nuevo orden patriarcal las mujeres están excluidas del trabajo asalariado y subordinadas a los hombres y al cuerpo proletario mecanizado. La caza de brujas cumplió, para Federici, esa función: las mujeres, bajo el control del Estado, fueron convertidas en recursos económicos para el orden patriarcal, les fue sustraído el control de la natalidad y su cuerpo fue puesto al servicio del incremento de la población y la acumulación de fuerza de trabajo. Si los marxistas latinoamericanos, como Aníbal Quijano, lograron mostrar lo intrínseco que es el racismo al capitalismo, Federici muestra el carácter patriarcal de la dominación capitalista. En ese sentido el "género", para la autora, es una especificación de las relaciones de clase.

La identificación del trabajo femenino como trabajo invisibiliza $\neg$ do tiene una relación directa con su condición de trabajo no pago en términos salariales. El trabajo de las mujeres queda minimizado como subsidiario del trabajo pago masculino y desconoce la vinculación entre ellos que, como sostiene Verónica Gago (2010), es intrínseca y su análisis, desde la perspectiva feminista, no introduce simplemente una especificidad o abona un particula $\neg$ rismo sino que abre la noción y la composición misma de la clase trabajadora, tanto en sus orígenes como en la actualidad.

De este modo, las teorías feministas, por un lado, dan cuenta del carácter estructural que tiene el rol subordinado de las mujeres y la división sexual del trabajo; por otro lado, analizan lo que ocurre con la explotación en el capitalismo actual. En ese sentido, Gago sostiene que la forma que adquiere el capitalismo neoliberal 
es la de producir nuevas com $\neg$ binaciones entre economías de tipo servil y economías posmodernas y que para eso no reproduce la tendencia hegemónica (o hegemonizante) de un trabajo asalariado libre, sino que lo hace a partir de la extensión de una nueva feminización del trabajo que implica la valo $\neg$ rización creciente de los atributos que permanentemente cualifican al trabajo como no-libre. Hay una con $\neg$ dición colonial de nuevo tipo, un nuevo impulso capitalista quelogra competitividad y dinamismo a partir de articularse con prácticas, redes y atribu $\neg$ tos que históricamente caracterizaron los flujos de trabajo no-pago, como lo fue siempre el trabajo de las mujeres. En ese sentido podemos afirmar que el rol del trabajo no-pago y el rol de las mujeres está en transformación en el modelo de acumulación actual, simultáneamente con una crisis económica mundial que ya lleva casi una década y que no ha cesado de profundizarse.

Respecto de los debates acerca de la categoría delgénero, Joan Scott (1990) sostiene queel género es un elemento constitutivo de las relaciones sociales basadas en las diferencias que distinguen los sexos $\mathrm{y}$, también, una forma primaria de relaciones significantes de poder. Comprende, según ella, cuatro elementos interrelacionados: símbolos culturalmente disponibles que evocan representaciones, múltiples (y menudo contradictorias), basadas en la diferencia; conceptos normativos que manifiestan las interpretaciones de los significados de los símbolos, en un intento de limitar y contener sus posibilidades metafóricas (doctrinas religiosas, educativas, científicas, legales y políticas, que afirman categórica y unívocamente el significado de varón y mujer, masculino y femenino); rol de las instituciones y organizaciones sociales en la construcción de una permanencia intemporal en la representación binaria del género (familia, mercado de trabajo, sistema educativo, política); y la identidad subjetiva.

Estos aportes muestran que, como sostiene Simone de Beauvoir (1949), mujer no se nace sino que se llega a serlo y articulan la desigualdad entre hombres y mujeres con el sistema global de dominación. Judith Butler (1990) hizo aportes significativos a la noción de género que, lejos de ser meras proposiciones teóricas o puntos de vista, han configurado acción política feminista y explican muchas de las posiciones que actualmente, en Argentina, se han volcado a hacer desde el feminismo una acción política específica sobre los regímenes de normatividad. La autora sostiene que el género es performativo, es decir que lo que muchas veces se considera esencia interna de género -nacer varón o nacer mujer en el cuerpo que sea- se construye a través de un conjunto sostenido de actos, postulados por medio de la estilización del cuerpo basada en el género. Yendo mucho más allá, sostiene también que aprender las reglas que rigen el discurso inteligible (donde sólo hay femenino y masculino, por ejemplo) es imbuirse del lenguaje normalizado, y el precio que hay que pagar por no conformarse a él es la perdida misma de inteligibilidad. Butler tiene como objetivo, entre otras cosas, poner en cuestión la mujer como sujeto del feminismo y discutir la noción de que exista un sujeto "antes" de la ley ("Lengua"), esperando su representación en y por ella.

Si el género es uno de los significados culturales que acepta el cuerpo sexuado, entonces no puede afirmarse que un género únicamente sea producto de un sexo. Si llevamos esta posición hasta su límite lógico, afirma Butler, la distinción sexo/genero muestra una discontinuidad radical entre cuerpos sexuados y géneros culturalmente construidos: no está claro que la construcción de "hombres" dará como resultado únicamente cuerpos masculinos o que las "mujeres" interpreten solo cuerpos femeninos. Para Butler, lo cuerpos no son binarios en su morfología y constitución, y tampoco hay ningún motivo para creer que los géneros seguirán siendo sólo dos. Butler sostiene una apuesta política contra el binarismo sexual y genérico: los géneros "inteligibles" son los que de alguna manera instauran y mantienen relaciones de coherencia y continuidad entre sexo, género y práctica sexual o deseo. Se presupone que la unidad metafísica de los tres, una visión sustancializadora del género. La desarticulación que propone Judith Butler entre sexo (o genitalidad), género $y$ deseo ha abierto un campo vasto de debates e identidades sexuales que, al concebirse como algo no sustancial sino performativo, $y$ 
que disputa con la llamada heterosexualidad normativa (que produce lo binario) viabiliza múltiples colectivos políticos que, efectivamente, quedan por fuera de la inteligibilidad femeninomasculino inscripta en la cultura $y$, en lo que a nosotros respecta, en las prácticas lingüísticas.

Entonces, como punto de partida, cualquier análisis de las prácticas lingüísticas es, necesariamente, una análisis de los regímenes de normatividad que produce y reproduce prácticas lingüísticas que garantizan relaciones de poder al interior de las cuales la división sexual del trabajo y la reproducción de las relaciones de desigualdad de género cumplen un rol estructurante. En el caso que analizamos, el régimen de normatividad entra en tensión cuando se pone en discusión una regla (también) estructurante del sistema lingüístico, como lo es la flexión genérica, por lo que produce reacciones vehementes, que van desde la desautorización hasta la violencia machista (insulto o acoso). Intentaremos demostrar que quienes, por un lado, desarrollan una práctica que enfrenta la norma o valoran positivamente esa práctica disruptiva y quienes, por el otro, participan de su censura y de su desprestigio, lo hacen según su posición en el entramado social y su nivel de subordinación ideológica al sistema de dominación que, como veremos en el apartado siguiente, se expresa en regímenes de normatividad y es reproducido por ellos.

\section{Algunos reflexiones en torno de la relación entre sexo, género, lenguaje y patriarcado}

Este apartado está lejos de ser un recorrido integral de aquellos autores que han pensado la relación entre estos cuatro elementos. El objetivo fundamental de este ensayo -muy acotado respecto de todas las dimensiones que implica el cruce entre lenguaje y patriarcado en términos amplios- se limita a ubicar esta reflexión en el terreno de la glotopolítica, y arrojar alguna hipótesis-desde esa perspectiva-en torno del uso del morfema " $\mathrm{e}$ " para el plural inclusivo. Hasta ahora hemos reflexionado en torno de una parte del problema. Para continuar, mencionaremos algunas aportaciones teóricas que, entre muchas otras que no están aquí, dan cuenta de la relación indiscutible entre la categoría gramatical de género -y su funcionamiento- y la existencia de una relación de desigualdad y dominación entre los hombres y el resto de las identidades.

Muchos manuales de lenguaje inclusivo sostienen sus propuestas de expresiones no sexistas sobre la base de que en el español existe una falta de representación simbólica de las mujeres en la lengua y que, por lo general, está invisibilizada. Aunque en el español sexo y género no coinciden en la mayoría de los casos, sí lo hacen en el caso de los sustantivos animados; por ende, la utilización del masculino, ya sea en singular para referirse a una mujer, o en plural para denominar a un grupo de mujeres o a un grupo mixto, según estos autores, esconde o invisibiliza a las mujeres, cuando no las excluye del proceso de representación simbólica que pone en funcionamiento la lengua (AAVV, 1995).

Respecto de tales planteos, Ignacio Bosque (2012), miembro de la Real Academia Española, sostiene que esa posición se encuentra con la pregunta de dónde fijar los límites ante el "problema de la visibilidad de la mujer en el lenguaje". Bosque se inquieta ante las propuestas porque sostiene, sobre la base de la propuesta Saussuriana, que si el sistema lingüístico da forma al pensamiento, es un sinsentido que algunas mujeres se sientan discriminadas. El segundo término, Bosque se inquieta por la dificultad para hablar, porque sostiene que las propuestas (que en los casos a los que se refiere son, fundamentalmente, las de reiteración - "los trabajadores y las trabajadoras"- y el reemplazo por palabras sin marca genérica -"la docencia" en lugar de "los docentes"-), puestas a funcionar de forma plena, impedirían hablar. El tercer elemento que inquieta a Bosque es el que se vincula con los tres rasgos correlativos de la Lengua que postula Saussure, y cuya determinación mutua resulta nodal para el pensamiento lingüístico tradicional: arbitraria - social - inmutable. Es decir, que la Lengua no es algo que se pueda poner en debate ya que se impone a los hombres más allá de su voluntad. Sin embargo, Bosque abre una puerta que tampoco Saussure cierra, y es que, aunque la arbitrariedad sea la norma, y el pensamiento 
mismo resulte moldeado por la lengua, en ciertos fenómenos gramaticales -como la existencia de dos géneros- puede encontrarse un sustrato social -es decir, sin arbitrariedad-. Sin embargo, lo más probable, dice Bosque tras su concesión, es que su reflejo sea ya "opaco" y que sus consecuencias en la conciencia lingüística de los hablantes sean "nulas".

Muchos lingüistas coinciden en este último punto, es decir, en la existencia de un sustrato no arbitrario en la categoría gramatical de género en los casos en los que coincide con el sexo. Para muchas corrientes de estudios lingüísticos provenientes de los estudios feministas, el lenguaje es androcéntrico (en estos casos se refieren tanto al nivel del sistema como al del enunciado). El androcentrismo puede identificarse, según Mercedes Bengoechea (2008), por dos rasgos: toda persona es del género masculino, a no ser que se especifique lo contrario (1) y las mujeres quedan borradas de la lengua (2). La autora aporta dos ejemplos que por su simpleza resultan elocuentes:

A. Llegaron los tres al pueblo

B. Los nómadas se trasladaban con sus enseres, mujeres y niños, siguiendo la caza.

El primer caso es un uso inclusivo del masculino como forma no marcada. La autora sostiene que el hecho de que podrían ser tres hombres o dos mujeres y un caballo prueba el principio que sostiene que toda persona es hombre a menos que se compruebe lo contrario. El segundo caso (que es un ejemplo común y podrían encontrarse ejemplos similares en la totalidad de la literatura de cualquier disciplina del mundo occidental) pretende ejemplificar la falacia de la inclusión, en la medida en que "los nómadas" - forma masculina que se supone que incluye a las mujeres- trasladaban objetos, entre los cuales había mujeres, lo que muestra que en "los nómades", $\mathrm{y}$, asimismo, en todos los usos del masculino inclusivo, la mujer no está incluida sino borrada.

Estas posiciones sostienen que la regla que supone que el masculino incluye al femenino no es de orden natural, eterno e inmutable, sino un claro reflejo de la visión androcéntrica del mundo y de la lengua. Según Bengoechea, la presencia constante de tales enunciados otorga carta de naturaleza a uno de los ejes vertebradores del androcentrismo, constante y habitual en la lengua: el que refuerza la presencia del género/sexo masculino y causa la desaparición del género/sexo femenino.

Cameron (1985), lingüista norteamericana que ahora produce desde la Universidad de Oxford, sostiene que lo que describe Bengoechea puede ser llamado "alienación" de las mujeres del lenguaje. Es decir, las palabras no les pertenecen $\mathrm{y}$, de algún modo, se les vuelven en contra. De este modo, la mirada feminista del lenguaje resulta una reminiscencia de la mirada feminista de la sexualidad: es una fuente poderosa que es apropiada por el opresor que devuelve simplemente la sombra que las mujeres necesitan para funcionar en la sociedad patriarcal. Desde este punto de vista, la lucha por el lenguaje resulta crucial en la lucha por la liberación de las mujeres de la situación de opresión.

Cameron aporta un recorrido histórico en la teoría feminista del lenguaje e identifica tres posiciones: los estudios de la diferencia sexual, centrados en estudiar si las mujeres y los hombres utilizan el lenguaje de forma diferente; los estudios de sexismo lingüístico, sus efectos y cómo eliminarlos $\mathrm{y}$, finalmente, aquellos que trabajan la dimensión de la alienación. La autora se pregunta si el lenguaje es el mecanismo por el cual la misoginia es construida y transmitida, y si acaso podemos pensar por fuera de los confines del lenguaje que odia a las mujeres. Recorre, en ese sentido, muchas posiciones que van desde las teorías del reflejo, hasta aquellas feministas que, ancladas en posiciones posestructuralistas, han reivindicado el relativismo o las posiciones de raigambre lacaniana. Su repaso por la teoría gramatical muestra que, a pesar de que algunos lingüistas insistan en demostrar la inexistencia de un vínculo entre género gramatical y sexo, tal vínculo resulta indiscutible. Para Cameron, sea cual fuere el origen del género gramatical, no pude afirmarse que no tiene que ver con el sexo en la medida en que es claro que se pone al servicio de los valores y las relaciones patriarcales. 
La autora afirma que aquello que sostiene Simone de Beauvoir (1949), acerca de la condición biológica innata de lo femenino y la configuración sociocultural de ser una mujer, está lexicalizado en el inglés a partir de "sexo" y "genero". Para las lingüistas feministas pioneras que investigaron el lenguaje y la genericidad a principios de la década del setenta, el objetivo fundamental era justamente, mostrar el modo en el que utilizar el lenguaje estaba implicado en el proceso de convertirse en una mujer o en un hombre. Es decir que utilizar un lenguaje androcéntrico -que asigna valor universal al hombre a partir del borramiento de la mujeres una parte del proceso sociocultural de convertirse en una mujer.

Butler -a quien retomamos luego- constituye, según Cameron, la respuesta posmoderna a ese debate, ya que sostiene que conocemos el "sexo" sólo a través del filtro ideológico del género que provee el discurso. De este modo, no hay uno más natural que el otro, ambos son, para Butler, construcciones culturales. La teoría de Bultler se basa en la obra de Jacques Lacan y de su concepción del sujeto como resultado de la entrada en un universo simbólico, su entrada en el lenguaje. Las normas de género -actos y gestos que nos esperan desde antes del nacimiento- son interpretados por Butler en términos similares al "orden simbólico" lacaniano. En ese sentido, el lenguaje es una estructura que está ya ahí, y que va a ser determinante en la producción de la subjetividad. Por eso los enunciados de género, desde los pronunciados en el nacimiento -"es un niño" o "es una niña"-, hasta los insultos como "maricón" o "marimacho" no son constatativos sino performativos, es decir, invocaciones o citaciones ritualizadas de la ley heterosexual (Senz, Preciado 1997)

Respecto de la gramática y el lenguaje inclusivo, Butler afirma lo siguiente:

Las formulaciones que tergiversan la gramática o que de manera implícita cuestionan las exigencias de la estructura de la lengua son claramente irritantes para algunos. Los lectores tienen que hacer un esfuerzo, y a veces estos se ofenden ante lo que tales formulaciones exigen de ellos. ¿Están los ofendidos reclamando de manera legítima un "lenguaje sencillo", o acaso su queja se debe a las expectativas de vida intelectual que tienen como consumidores? ¿Se obtiene, quizá, un valor de tales experiencias de dificultad lingüística? Si el género mismo se naturaliza mediante las normas gramaticales, como sostiene Monique Wittig, entonces la alteración del género en el nivel epistémico mas fundamental estará dirigida, en parte, por la negación de la gramática en la que se produce el género. No estoy fuera del lenguaje que me estructura, pero tampoco estoy determinada por el lenguaje que hace posible este "yo". Este es el vínculo de autoexpresión, tal como lo entiendo. Lo que significa que usted, lectora o lector, no me reciben nunca separada de la gramática que permite mi disponibilidad con usted. Si trato esa gramática como algo de claridad meridiana, entonces no podre despertar su interés por esa esfera del lenguaje que establece y desestablece la inteligibilidad (Butler 1990).

Regresamos, desde la perspectiva de Butler, a pensar el problema de la representación y la visibilidad con el que discutía Bosque, desde la razón de la Real Academia Española. Según la autora, para las teorías feministas, el desarrollo de un lenguaje que represente de manera adecuada y completa a las mujeres fue necesario para promover su visibilidad política. Es decir qué representación política estuvo asociada a la noción de representación en el signo. ¿Pero qué ocurre con aquellos a los que la lengua no representa? ¿Para quienes no existe signo? La noción (androcéntrica) del signo masculino como universalidad supone un sentido de universalidad que incluye el binarismo o la ley heterosexual. Si no hay más dos sexos, y si no hay signo para representar en el lenguaje como acción política- aquello que pelea por una representación política, entonces hay que desplazarse a los márgenes, a lo que esté cerca de perder inteligibilidad.

En La Dominación Masculina Bourdieu (1998) también reflexiona, acerca del carácter no natural de la diferencia sexual y, a su vez, del rol que cumplen algunas estructuras simbólicas -como el lenguaje- en la reproducción de relaciones de dominación. Para el autor las necesidades de la reproducción biológica no son las que determinan la organización 
simbólica de la división sexual del trabajo y, progresivamente, de todo el orden natural y social -es decir no hay dos sexos cuya diferencia sea la causa de dos formas de representación simbólica del sexo en, por ejemplo, el género gramatical. Más bien es una construcción social arbitraria de lo biológico, y en especial del cuerpo, masculino y femenino, de sus costumbres y de sus funciones, en particular de la reproducción biológica, que proporciona un fundamento aparentemente natural a la visión androcéntrica de la división de la actividad sexual y de la división sexual del trabajo y, a partir de ahí, de todo lo demás. "La fuerza especial de la sociodicea masculina procede de que acumula dos operaciones: legitima una relación de dominación inscribiéndola en una naturaleza biológica que es, en sí misma, una construcción social naturalizada" (1998: 38). Las inclinaciones, es decir, el habitus no puede pensarse por separado de aquellas estructuras que lo producen y lo reproducen.

Todas estas reflexiones nos permiten ver que la estructura de la lengua y el valor universal $o$ inclusivo del género gramatical masculino tienen una relación indiscutible con el género como categoría sociocultural -y, para algunos autores, con el sexo- y con el funcionamiento de las relaciones de poder en la sociedad. En primer lugar, lo femenino está borrado o está subordinado en la estructura de la lengua, al igual que todo aquello que queda por fuera de la norma heterosexual -como expresa Butler- o del binarismo sexual -como diría Bourdieau-. Lo masculino es universal, lo femenino esta borrado y lo que no es femenino o masculino queda en los límites de lo inteligible, no tiene representación. Las teorías feministas han logrado demostrar, como vimos en el apartado anterior, que lo mismo ocurre en la estructura social respecto del rol asignado a varones y mujeres -y otras identidades- y el rol fundamental que cumplen en sistema de acumulación. Las prácticas lingüísticas de los sujetos, es decir, la disposición a utilizar y valorar la lengua de cierto modo - habitus- dependerán, como decía Del Valle, del grado de sometimiento o autonomía con respecto al régimen de normatividad imperante estructurado por esas condiciones sociales y relaciones de poder. El rol de los regímenes de normatividad en la reproducción social es el aspecto más relevante de este debate, incluso por encima de los debates acerca de si la existencia de dos géneros es o no la codificación en la lengua -que así planteado sería una entidad que puede pensarse por fuera de las prácticas lingüísticasde una diferencia sexual preexistente.

\section{En nombre de La Lengua}

Ferdinand de Saussure (1916) es considerado el "padre" de la lingüística moderna y configuró un objeto de estudio hasta entonces inexistente, a partir de un punto de vista sincrónico, redefiniendo los parámetros del pensamiento gramatical europeo $\mathrm{y}$, por su influencia, del mundo: La Lengua. Según Saussure, la lengua tiene, entre otros, dos principios fundamentales que son los que, en este caso, están puestos en cuestión y son la respuesta inmediata de muchos lingüistas y filólogos al planteo de las corrientes que pretenden promover el lenguaje inclusivo: su arbitrariedad y su inmutabilidad -sincrónica-. Las consecuencias de la arbitrariedad, sostiene Saussure, son incalculables, y, justamente, la inmutabilidad es una de ellas, una de las más importantes. La lengua no pone nombres al mundo, sino que es, en sí misma un principio de clasificación, la aparición de lo distinto: la lengua da forma al pensamiento porque provee la posibilidad de que haya un sonido distinto de otro e inmediatamente unido a un concepto -por primera vez distinto de otro-. En ese sentido, las categorías gramaticales de género -femenino y masculino- de la lengua no se consideran asimilables al sexo, sino que son pensadas como algo autónomo provisto por ella y -esto es lo más importante de todo- por esa inmotivación indiscutible, no se pueden modificar. Incluso podemos afirmar que, para Saussure, la mutabilidad diacrónica está teóricamente subordinada a la misma arbitrariedad, porque es esa permanencia inmodificable en el tiempo la que hace que esta cambie sin que esos hablantes lo noten. En este punto resulta fundamental recordar un principio contradictorio pero necesario para pensar la lógica saussuriana: teóricamente no existe habla sin Lengua, pero históricamente, el habla precede siempre. 
Voloshinov elaboró una crítica profunda a lo que llamó el "objetivismo abstracto" de Saussure. La perspectiva de Voloshinov llegó a ser conocida a través de la teoría de los géneros discursivos y de dialogismo (y la polifonía), atribuidas a Mijail Bajtin (1952-1953) ${ }^{6}$. Sin embargo, el carácter materialista y la radicalidad de la crítica de Voloshinov fue mayormente invisibilizada y subestimada, tanto desde las posiciones estructuralistas y posestructuralistas como desde las perspectivas del materialismo determinista, para quienes los aportes de Voloshinov también se alejaban fuertemente de la teoría del reflejo. Sin embargo, lo que aporta es, justamente, una de las claves para pensar los conflictos en torno del significado del morfema del masculino en el español: el signo es la arena de la lucha de clases, esto es, de la lucha ideológica. Es decir, la clase dominante busca adjudicar al signo ideológico un carácter eterno por encima de las clases sociales, pretende apagar y reducir al mínimo la lucha de valoraciones sociales que se verifica en él, trata de convertirlo en un signo monoacentual. Así es como se plantea, para Voloshinov, la relación entre las bases y las superestructuras: sin determinación mecánica. Si el lenguaje expresa, de algún modo, la superestructura de una sociedad, lo hace en función de las relaciones materiales (de explotación, por ejemplo) bajo las cuales se organiza. En este sentido la lengua no refleja sino que refracta y el significado de los signos puede estar en disputa, del mismo modo que el resto de las relaciones sociales.

Para Voloshinov, la teoría Saussuriana es un objetivismo abstracto por dos razones, en primer lugar porque niega el rol de la subjetividad, y, en segundo lugar, porque para hacerlo imagina situaciones que no ocurren en la realidad, ejecuciones individuales de un sistema de la lengua que no ocurren jamás por fuera de experiencias concretas históricamente situadas, de sujetos reales que toman la palabra y producen enunciados orientados. En ese sentido, la oposición entre social e individual -de la que surgen otras dicotomías- es problemática teóricamente y es la que funda el objeto de estudio y sostiene las nociones de mutabilidad e inmutabilidad. Para Voloshinov, en la propuesta Saussuriana, no hay ninguna relación entre la lógica del lengua como sistema de formas (sincronía) y la lógica de su generación histórica (diacronía). En ambas esferas predominan leyes toralmente diferentes, pues aquello que hace a la unidad y la conciencia de la lengua en su corte sincrónico, se transgrede y se subestima en el corte diacrónico. Para Voloshinov, la propuesta de Saussure supone que el presente de una lengua y su historia no son capaces de comprenderse una a otra. De esa reflexión es que concluye que el acto individual del habla, de la enunciación, minimizado y denostado en la lingüística, regresa sin embargo como el factor necesario de la historia de la lengua.

La distinción entre diacronía y sincronía es, para el autor, el resultado de un punto de vista elaborado desde la filología, que trabajaba con lenguas muertas. El enunciado aislado, acabado y monológico, sacado de su contexto discursivo real, no orientado hacia una posible respuesta activa, sino a la comprensión pasiva de un filólogo, representa el dato último y el punto de partida para el pensamiento lingüístico: la Lengua para la lingüística está muerta, es escritay, fundamentalmente, es ajena. Voloshinov revierte esa mirada y sostiene que nada existe realmente para la conciencia subjetiva del hablante como un sistema objetivo de incuestionables formas normativamente idénticas. Lo que al hablante le importa es aquel aspecto dela forma lingüística gracias el cual resulta un signo apropiado para las condiciones concretas de una situación dada: en la vida real no pronunciamos ni oímos palabras, sino que oímos la verdad o la mentira, lo bueno o lo malo, lo importante o lo nimio, lo agradable o lo desagradable. La palabra siempre aparece llena de un contenido, o de una significación ideológica o pragmática.

6 Hay muchos debates en este punto. Algunos sostienen que eran personas distintas pero que reflexionaban en conjunto desde el llamado Círculo de Bajtín. Otros sostienen que se trata de la misma persona. No nos interesa posicionarnos sobre este punto. 
En resumen, podemos afirmar que para la lingüística que funda el pensamiento saussuriano, el momento estable e idéntico a si mismo de las formas lingüísticas (sincronía) prevalece sobre su variabilidad (diacronía); lo abstracto prevalece sobre lo concreto y la sistematicidad abstracta prevalece sobre la historicidad -lo que no permite anclarlo históricamente; las formas de los elementos prevalecen sobre las formas de la totalidad y la sustancialización del elemento lingüístico aislado sustituye la dinámica del discurso- es decir, asistimos a una historia de las formas, y no a una historia de los enunciados reales producidos por sujetos reales en su experiencia vital -la monosemia y la monoacentualidad de la lengua sustituye su polisemia y su poliacentualidad- es decir, al anularse la dimensión viva y dinámica, se anula el punto de vista del conflicto $y$, finalmente, se presenta el concepto de La Lengua como una entidad abstracta y acabada que se transmite de una generación a otra. Queda afuera cualquier posibilidad de una participación activa de una conciencia hablante en el proceso de la generación histórica. Es inviable cualquier combinación dialéctica entre la necesidad y la responsabilidad lingüística o la libertad. Asumir el punto de vista de La Lengua supone, entonces, pararse desde un objetivismo abstracto desprovisto de historicidad.

En el sentido de esta crítica materialista y desde la perspectiva del conflicto es que nos permitimos pensarel problema dela aparición del morfema " $\mathrm{e}$ " como flexión de género inclusivo. En primer lugar, si el lenguaje (en tanto régimen de normatividad) refracta las características de la estructura social, tiene mucho sentido, también en términos de Voloshinov, que el masculino sea, por defecto, lo universal y que ese sea el sentido que se le quiera dar desde aquellos sectores interesados en la reproducción de las actuales relaciones de poder. En segundo lugar, si el signo es uno de los lugares en los cuales se despliega la lucha de clases -en forma de batalla ideológica- creemos que el fenómeno a atender no es solamente la aparición de la " $\mathrm{e}$ " y las posibilidades de planificar y de tener éxito -o noen la introducción de un cambio lingüístico tan significativo, sino más bien la batalla ideológica abierta acerca de la universalidad del masculino.
En ese sentido, la aparición de otro significante para expresar la inclusión de todas las identidades, o bien de aquellas para las que no existe una forma estable, es una consecuencia de que el significado del masculino esté en disputa. El significado universal y genérico del masculino está tan en crisis como lo está el rol social de poder de los hombres en la sociedad patriarcal, producto, tanto de una transformación de algunos elementos del sistema de acumulación, como de la lucha del movimiento feminista en el mundo. Como sostiene Voloshinov, en la palabra se ponen en funcionamiento los innumerables hilos ideológicos que traspasan todas las zonas de la comunicación social. Es un indicador sensible de las transformaciones sociales, inclusive aquellas que apenas van madurando $\mathrm{y}$ aún no se constituyen ni encuentran acceso todavía a los sistemas ideológicos ya formados y consolidados.

\section{Conclusiones}

Este ensayo está atravesado por un conflicto fundamental: no tiene del todo claro quiénes son sus destinatarios. No es lo mismo escribir para los lingüistas que para las feministas. Parece innecesario aclarar que los lingüistas no son todos varones cis. Más innecesario todavía pareciera ser tener que aclarar que las feministas no son todas mujeres cis; sin embargo, decir "los feministas" es casi un contrasentido -por no decir un acto de violencia-. El conflicto parte de una situación que se sostiene en la mayoría de los ámbitos de producción de conocimiento: este es un debate lingüístico y político -si es que existe alguno lingüístico que no lo sea- que no puede darse sin leer la producción de los feminismos, tanto de teoría lingüística como de otros campos. Los lingüistas no se han nutrido lo suficiente de las teorías feministas y poco tienen para aportar a este debate si no conocen la mitad del problema.

El fenómeno de los adolescentes hablando con " $\mathrm{e}$ ", pero fundamentalmente el fenómeno de un movimiento feminista masivo y plural (construido con un siglo de luchas, reflexiones $\mathrm{y}$, sobre todo, silenciamientos teóricos $\mathrm{y}$ políticos) ha puesto a los lingüistas en un brete. 
La sociedad insiste en posicionarse acerca del posible o imposible cambio lingüístico, acerca del cual la corporación lingüística responde, en nombre de "La Lengua", que esa transformación voluntaria no es posible. La pregunta no parece residir en si Saussure estaba o no en lo cierto: aunque los fundamentos de la inmutabilidad de la Lengua han permitido niveles muy profundos de comprensión del fenómeno lingüístico, son discutibles desde varios aspectos. Pensar este problema supone desplazarnos de un debate acerca de la Lengua -como entidad abstracta que cuya definición la contrapone al habla, es decir, al uso- para asumir un debate acerca de las prácticas lingüísticas y los regímenes de normatividad. La pregunta, en todo caso, es, en primer lugar, de qué modo las prácticas lingüísticas integran los mecanismos de reproducción de las relaciones de poder y dominación y cómo las sociedades se dan estrategias, más o menos efectivas, para poner en tensión esas relaciones; y en segundo lugar, qué transformaciones económicas, sociales y políticas están en curso que impactan en el lenguaje (refractan, en términos de Voloshinov) $\mathrm{y}$, a su vez, permiten niveles de conciencia e insubordinación de colectivos tan amplios a los regímenes de normatividad vigentes.

A partir de un recorrido teórico que, insistimos, es exploratorio y pretende profundizarse, consideramos que, en primer lugar, cualquier análisis de las prácticas lingüísticas no sexistas o de lenguaje inclusivo exige un análisis de los regímenes de normatividad que produce y reproduce prácticas lingüísticas que garantizan relaciones de poder al interior de las cuales la división sexual del trabajo y la reproducción de las relaciones de desigualdad de género cumplen un rol estructurante. La sociedad es muy reactiva -con insultos, indignación, desautorización, violencia- cuando se pone en discusión una regla fundamental del sistema lingüístico (la flexión genérica) porque hay una insubordinación al régimen de normatividad vigente $\mathrm{y}$, por ende, a las relaciones de poder que lo estructuran. Las prácticas lingüísticas de los sujetos (o bien las valoraciones acerca de ellas) dependen del grado de sometimiento o autonomía con respecto a esos regímenes.
En segundo lugar, a partir de un recorrido por algunos planteamientos teóricos, hemos visto que hay muchos elementos para considerar que la estructura de la lengua y el valor universal o inclusivo del género gramatical masculino tienen una relación con el género como categoría sociocultural $-\mathrm{y}$, para algunos autores, con el sexo- y con el funcionamiento de las relaciones de poder en la sociedad. Por un lado, lo femenino está borrado / subordinado en la estructura de la lengua $-y$ aquello que no reproduzca la norma heterosexual que queda en los límites de lo inteligible y no tiene representación- al igual que en la estructura social.

Por último, si analizamos el nivel del sistema de la lengua -que, en términos de Voloshinov, tampoco existe como entidad abstracta por fuera de un uso social concreto-, este refracta las características de la estructura social y el masculino resulta, por defecto, lo universal. Ese es el significado que asumen como el único posible desde aquellos sectores implicados en la reproducción de las actuales relaciones de poder. En ese sentido, el fenómeno a atender no es solamente la aparición de la " $\mathrm{e}$ " y las posibilidades de planificar y de tener éxito -o noen la introducción de un cambio lingüístico tan significativo, sino más bien la batalla ideológica abierta acerca de la universalidad del masculino. La aparición de otro significante para expresar la inclusión de todas las identidades, o bien de aquellas para las que no existe una forma estable, es una consecuencia de que el significado del masculino esté en disputa, al igual que lo está el rol social de poder de los hombres en la sociedad patriarcal.

\section{Referencias bibliográficas}

Alario, C. - Bengoechea, M. - Llendó, E.- Vargas, A. 1995. La representación de lo femenino y de lo masculino en la lengua. Madrid: Instituto de la Mujer.

Arnoux, E. 2000. “La Glotopolítica: transformaciones de un campo disciplinario". En Lenguajes: teorías y prácticas. Buenos Aires: Instituto Superior del Profesorado "Joaquín V. González", Secretaría de Educación, GCBA, s/p. 
Arnoux, E. 2008a. Los discursos sobre la nación y el lenguaje en la formación del Estado (Chile, 1842-1862). Estudio glotopolítico. Buenos Aires: Santiago Arcos.

Arnoux, E. y J. Del Valle 2010 "Las representaciones ideológicas del lenguaje. Discurso glotopolítico y panhispanismo", en Elvira Arnoux y José del Valle, Spanish in Context, número especial sobre "Ideologías lingüísticas", John Benjamins.

Bengoechea, M. 2008 .“Guía para la revisión del lenguaje desde la perspectiva de género", Madrid: Comisión de utilización no sexista del lenguaje y de la imagen.

Bosque, I. (4 de marzo de 2012). "Sexismo lingüístico y visibilidad de la mujer", Madrid: El País.

Bourdieu, P. 1998. La Dominación Masculina, Barcelona: Anagrama.

Butler, J. 1990. El género en disputa. El feminismo y Ia subversion de la identidad, México: Paidós.

Cameron, D. 1985. Feminism and Linguistic Theory, London: The Macmillan Press.

De Beauvoir, S. 1949 (2015) El Segundo Sexo, Madrid: Cátedra.
De Saussure, F. 1916 (1993) Curso de Lingüística General, Buenos Aires: Planeta Agostini.

Federici, S. 2004 (2016) Calibán y la Bruja. Mujeres, cuerpo y acumulación originaria, Buenos Aires: Tinta Limón.

Gago, V. 2010. La Razón Neoliberal. Economías Barrocas y Pragmática Popular, Buenos Aires: Tinta Limón.

Laroussi, C. 2003. “Quelle politique linguistique pour quel Etat-nation?". En Glottopol, n 1 .

Marcellesi, J.B. y Guespin, L. 1986. “Pour la glottopolitique", en Revista Langages, $\mathrm{N}^{\circ}$ 83, pp. 5-34.

Minici, F. 2018. "Resistencia permanente", en Malena Nijensohn (comp), Los feminismos ante el neoliberalismo, Buenos Aires: Latfem / Ediciones La Cebra.

Saenz, J - Preciado, B. 1997. “Prólogo" en Judith Butler, Lenguaje, Poder e Identidad, Madrid: Síntesis.

Scott, J. 1990. "Gender: A Useful Category of Historical Analysis" en American Historical review, 91,1986, pp. 1053-1075.

Voloshinov, V. 1929 (2009) El Marxismo y la Filosofía del Lenguaje, Buenos Aires: Ediciones Godot. 


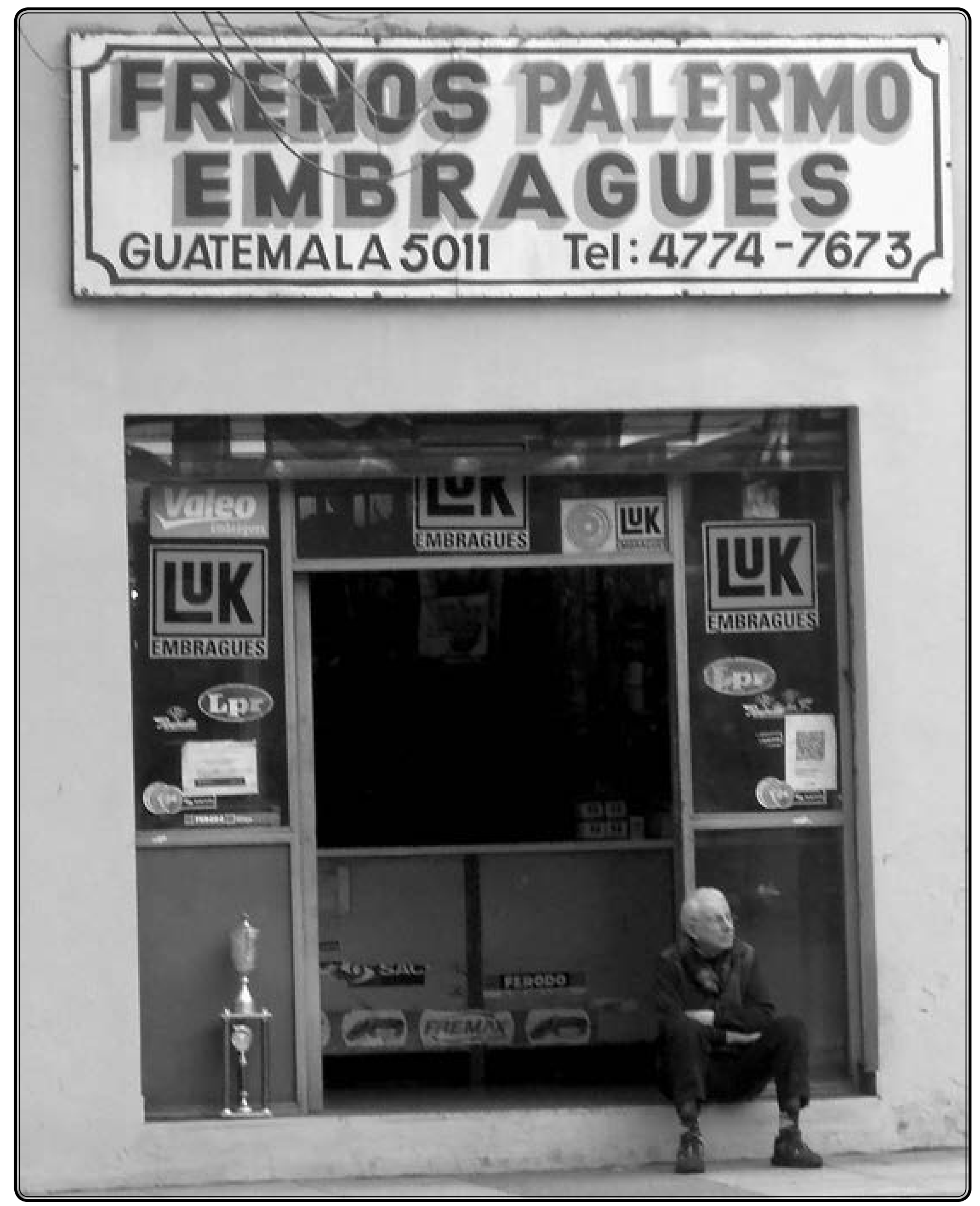

Figuritas de antes. 